\title{
Comparative Response of Newtonian and Non-Newtonian Fluids Subjected to Exothermic Reactions in Shear Flow
}

\section{Tiri Chinyoka ${ }^{1}$ (D)}

Accepted: 19 April 2021 / Published online: 24 April 2021

(c) The Author(s), under exclusive licence to Springer Nature India Private Limited 2021

\begin{abstract}
A comparative computational study of the thermal response of Newtonian and non-Newtonian fluids subjected to exothermic reactions is conducted in simple shear flow. The investigations conducted in this study are of fundamental importance to industrial and biological applications in which heat generation minimization is important, such as in heat exchangers, in lubrication, and in internal medicine. Specifically, the comparative investigations central to this study are conducted on four types of fluids, namely; Newtonian fluids, generalized Newtonian fluids, viscoelastic fluids, and generalized viscoelastic fluids. The Oldroyd-B constitutive model is used for the viscoelastic fluids and a Carreau viscosity constitutive model is used to describe the viscosity shear-rate dependence of the generalized fluids. Efficient semi-implicit finite difference methods are employed to obtain the numerical solutions to the governing systems of equations. The computational methodologies are implemented in the MATLAB software. The sensitivity of the fluid temperature and the polymer stresses to increases in shear-thinning characteristics as well as to increases in polymeric properties is investigated. In general, it is observed that, at comparative parameter values, the viscoelastic fluids give the best resistance to temperature increases, followed by the generalized viscoelastic fluids, followed in turn by the Newtonian fluids, and with the generalized Newtonian fluids recording the highest temperature increases.
\end{abstract}

Keywords Newtonian and non-Newtonian fluids · Exothermic reactions · Shear flow · Finite difference methods

Mathematics Subject Classification 35Q35 · 76M20

\section{Introduction}

Fluids in general make life possible. The air we breath, the water we need to sustain life are examples of fluids. The specific focus on non-Newtonian fluids also finds wide application and occurrence in everyday life. Such applications and occurrences range from natural phenomena

Tiri Chinyoka

tchinyok@vt.edu

1 Center for Research in Computational and Applied Mechanics, University of Cape Town, Rondebosch 7701, South Africa 
to human induced activity and processes. Naturally occurring phenomena which exhibit nonNewtonian fluids and flows include mud-slides and magma flows. Non-Newtonian fluids and flows permeate virtually all aspects of human activity including, in household products such foodstuffs, toiletries, and pharmaceuticals; in mining and industrial processes such as petroleum exploration and transport; in biological processes such as blood flow and internal medicine; etc. Investigations into the heat transfer characteristics of fluids are of fundamental importance in a wide range of industrial processes, say in energy generation, heating and cooling applications, lubrication, etc. see for example [1] for applications to heat exchanger efficiency and [2] for applications to lubricant design.

For a comprehensive account of the historical perspectives on the mechanics and flow of non-Newtonian fluids, the reader is referred, say, to the works in [3-11]. Non-Newtonian fluids can be classified into two broad categories depending on whether or not they exhibit elastic characteristics. Elastic behaviour is a characteristic of polymeric fluids, such fluids are generally referred to as viscoelastic fluids essentially because they posses both viscous and elastic properties. Viscous and inelastic non-Newtonian fluids are distinguished from Newtonian fluids by the shear-rate dependence of their viscosities. Such viscous and inelastic fluids are generally referred to as Generalized Newtonian Fluids. Fluids can therefore be classified into four broad categories as follows.

Newtonian fluids. These fluids do not possess any elastic properties and they exhibit constant shear-viscosity in viscometric experiments. Newtonian fluids are therefore inelastic fluids whose viscosities do not depend on shear-rates. Any fluid whose glow behaviour can be described according to the Navier-Stokes equations is by definition a Newtonian fluid!

Generalized Newtonian fluids. These fluids also do not possess any elastic properties but they exhibit variable shear-viscosity in viscometric experiments. Generalized Newtonian fluids are therefore inelastic fluids whose viscosities depend on shear-rates. The description of Generalized Newtonian fluids in this paper will therefore be restricted to the description of the shear-rate dependent viscosity. In particular, Carreau viscosity constitutive model will be used to describe the viscosity shear-rate dependence of the Generalized Newtonian fluids.

Boger fluids. Boger fluids are elastic fluids whose viscosities do not depend on shearrates. As with Newtonian fluids, Boger fluids exhibit constant shear-viscosity in viscometric experiments. Because they possess both viscous and elastic properties, Boger fluids are referred to a viscoelastic fluids. Reference to viscoelastic fluids in this paper will therefore be synonymous with Boger fluids. The Oldroyd-B fluid model will be used as the illustrative example of a Boger fluid in this paper.

Generalized Viscoelastic fluids. Generalized Viscoelastic fluids are viscoelastic fluids whose viscosities are shear-rate dependent. The Generalized Viscoelastic fluids therefore combine the properties of viscoelastic fluids (i.e. fluid elasticity) and Generalized Newtonian fluids (i.e. Carreau viscosity model). The Generalized Oldroyd-B fluid model will be used as the illustrative example of a Generalized Viscoelastic fluid in this paper.

In this paper, a comparative analysis of the response of these four categories of fluids to non-isothermal effects and exothermic reactions in simple shear flow is conducted. Attention is limited to shear-thinning behaviour in modelling the shear-rate dependence of viscosity. In [2], it was demonstrated that Newtonian fluids are much more susceptible to thermal runaway phenomena (finite time temperature blow up) as compared to viscoelastic fluids. Additionally it was also demonstrated in [2] that the more elastic a fluid becomes, the less susceptible it became to thermal runaway phenomena. Similarly, the exploratory study in [12] demonstrated that strongly shear-thinning Generalized Newtonian Fluids are more susceptible to thermal runaway. The exothermic studies are therefore of great importance in view of physical applications. In addition to the examples just cited, all four categories of fluids 


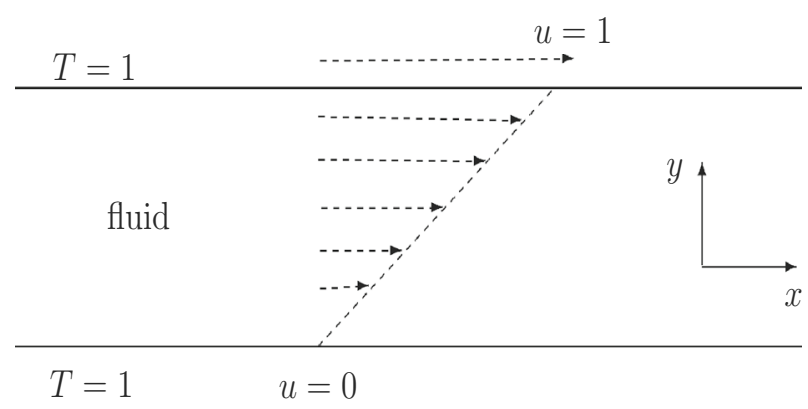

Fig. 1 Schematic of the model problem

have been used in blood flow simulation studies. The respiratory processes in blood cells can lead to anomalous exothermic heat release processes, especially under conditions of fever based ailments such as Covid-19. The understanding (and indeed comparative analysis) of the response of these four categories of fluids to non-isothermal effects and exothermic reactions is therefore of fundamental contemporary significance.

Temperature and/or concentration effects in flow of non-Newtonian fluids have indeed received much attention over the years, see for example [13-16]. The flow equations of Newtonian and non-Newtonian fluids form nonlinear systems of coupled partial differential equations which are not amenable to analytic solution methodologies. The added complexities arising from the temperature effects only compounds these problems. It is therefore inevitable that a recourse be made to numerical solution methodologies and related computational techniques. To this end, a variety of computational methodologies have been deployed for non-Newtonian flow problems, see for example [17-20].

\section{Mathematical Modelling}

The schematic of the model problem is given in Fig. 1. An incompressible fluid is subjected to shear flow between two infinite parallel plates. The $x$-axis is positioned parallel to the direction of motion and the $y$-axis normal to it. The solid bounding plates are maintained at the constant temperature $T_{w}$ and that the fluid is subjected to Arrhenius type exothermic reactions. The fluid motion is driven entirely by the motion of the upper plate. The fluid is viscous and hence the boundary conditions for the velocity derive from the no-slip conditions at the solid walls.

\section{Why Conduct This Study?}

Investigations into the heat transfer characteristics of fluids are of fundamental importance in a wide range of industrial and biological processes. For example;

(i) In heat exchangers (say with applications to food processing, etc.) an important question relates to which fluid arrangements would give the most efficient heat exchange characteristics?

(ii) In lubrication applications, which composition of the fluid lubricants would be least susceptible to thermal runaway phenomena? 
(iii) In blood flow the correct material modelling would be fundamental in understanding and intervening on fever based ailments that may be exacerbated by the exothermic respiratory processes in blood cells, etc.

(iv) The main fundamental question that is central to all such applications therefore reduces to; how does the specific Newtonian or non-Newtonian fluid character under conditions of heat release such as in exothermic reactions affect the flow application?

This paper provides the answers to these pertinent questions and issues.

\section{Dimensionless Equations}

The flow of Newtonian and non-Newtonian fluids, with the non-Newtonian models comprising both the Generalized Newtonian Fluid model as well as the viscoelastic fluid model is investigated. The fluid is thus incompressible and the flow system is governed by the equations of conservation of mass, momentum, and energy. In non-dimension form, see for example [20], the governing equations are given as,

$$
\begin{aligned}
\nabla \cdot \boldsymbol{u} & =0, \\
\operatorname{Re} \frac{D \boldsymbol{u}}{D t} & =-\operatorname{Re} \nabla p+\nabla \cdot\left(\boldsymbol{\tau}+2 \eta_{s} \boldsymbol{S}\right), \\
\operatorname{Re} \operatorname{Pr} \frac{D T}{D t} & =\nabla^{2} T+\operatorname{Br} Q_{D}+\delta_{1} \exp \left(\frac{T}{1+\alpha T}\right),
\end{aligned}
$$

where $\boldsymbol{u}=(u(t, y), 0)$ is the dimensionless velocity, $\boldsymbol{S}=\left[\nabla \boldsymbol{u}+(\nabla \boldsymbol{u})^{T}\right] / 2$ is the rate of deformation tensor, $T$ is the dimensionless temperature, $\boldsymbol{\tau}$ is the dimensionless extra stress (polymeric stress) tensor for viscoelastic fluids, Re is the Reynolds number, Pr is the Prandtl number, $\mathrm{Br}$ is the Brinkman number, $\delta_{1}$ is the Frank-Kamenetskii parameter, $t$ is the dimensionless time, $\boldsymbol{x}=(x, y)$ are the dimensionless coordinates in the longitudinal and transverse direction respectively, and $D / D t$ is the material derivative.

The extra stress tensor $\tau$ is specified by the Oldroyd-B or the Generalized Oldroyd-B constitutive model,

$$
\boldsymbol{\tau}+\operatorname{De} \bar{\lambda}\left(\stackrel{\nabla}{\boldsymbol{\tau}}-\boldsymbol{\tau} \frac{D}{D t} \ln (1+\alpha T)\right)=\eta_{p} \boldsymbol{S},
$$

where

$$
\underset{\boldsymbol{\tau}}{\nabla}=\frac{D}{D t} \boldsymbol{\tau}-\nabla \mathbf{u} \cdot \boldsymbol{\tau}-\boldsymbol{\tau} \cdot(\nabla \boldsymbol{u})^{T} .
$$

The dimensionless dissipation function, $Q_{D}$, is defined as,

$$
Q_{D}=2 \eta_{s} \boldsymbol{S}: \nabla \boldsymbol{u}+\gamma \boldsymbol{\tau}: \boldsymbol{S}+(1-\gamma) \frac{\hat{G}}{2 \operatorname{De} \bar{\lambda}(T)}\left(I_{1}+\operatorname{Tr}\left(\boldsymbol{b}^{-1}\right)-6\right),
$$

where $\hat{G}$ is the shear modulus, $\bar{\lambda}$ is the temperature constitutive model for the relaxation time and the colon : denotes the double dot product of two tensors. Unlike inelastic fluids (i.e. Newtonian and Generalized Newtonian Fluids) viscoelastic fluids can store energy due to their elastic properties. The parameter $\gamma$ with $0 \leq \gamma \leq 1$ is included to model this energy storing ability of viscoelastic fluids. The case $\gamma=1$ corresponds to pure entropy elasticity in which, as with inelastic fluids, the internal heat production reduces to the work done by the stresses. The case $\gamma=0$ on the other hand corresponds to energy elastic heat dissipation. 
Values of $\gamma$ in the range $0<\gamma<1$ correspond to linear combinations of stress work and dissipation. The conformation tensor $\boldsymbol{b}$ is related to the extra stress tensor $\boldsymbol{\tau}$ by,

$$
\boldsymbol{\tau}=\hat{G}(\boldsymbol{b}-\boldsymbol{I}),
$$

where $\boldsymbol{I}$ is the unit tensor. The temperature dependence of the relaxation time is given by,

$$
\bar{\lambda}(T)=\frac{1}{1+\alpha T} \exp (-\alpha T)
$$

where $\alpha$ is a dimensionless non-isothermal parameter.

As in Makinde et. al [21,22] it may be useful in the future to investigate extensions of the current work to include both flows whose shear-rates are non-constant at steady state as well as flows with non-trivial convective heat exchange characteristics. Such extensions will necessarily lead to physically important (and physically measurable) dimensionless groups such as the skin friction and the Nusselt number. In the current framework, however, the skin friction in steady state remains constant at $C_{f} \equiv 1$ (given the steady linear velocity profile) and the Nusselt number is also trivial at $N_{u} \equiv 0$, given the absence of convective heat transfer effects.

\section{Viscosity Constitutive Modelling}

The temperature dependence of the viscosities is given by a Nahme-type law,

$$
\mu(T)=\exp (-\alpha T)
$$

\section{Newtonian Fluids}

For Newtonian fluids ( $\boldsymbol{\tau}=\mathbf{0}$ ) only the temperature dependence of viscosity is important. In particular, the viscosity does not depend on shear-rates within the flow field nor is there any polymer contribution to the viscosity,

$$
\eta_{s}=\mu(T), \quad \eta_{p}=0 .
$$

\section{Generalized Newtonian Fluids}

For Generalized Newtonian fluids $(\boldsymbol{\tau}=\mathbf{0})$ both temperature dependence and shear-rate dependence of viscosity are important,

$$
\eta_{s} \rightarrow \eta(\dot{\gamma}) \mu(T), \quad \eta_{p}=0
$$

where, $\dot{\gamma}$ is the shear-rate,

$$
\dot{\gamma}=\sqrt{2 S: S},
$$

and the symbol $\rightarrow$ is used in Eq. (11) to indicate that the occurrence of $\eta_{s}$ should be replaced with a non-Newtonian viscosity $\eta$. This is done to avoid abuse of notation given that the solvent viscosity $\eta_{s}$ must always be used synonymously with Newtonian viscosity!

The shear-rate dependence of viscosity is modelled via the Carreau model, see for example Koriko et. al [23] and Makinde et. al [24],

$$
\eta(\dot{\gamma})=1+(m-1)\left(1+(\lambda \dot{\gamma})^{2}\right)^{\frac{n-1}{2}} .
$$


Attention is limited to shear-thinning viscosity in which $0 \leq n \leq 1$ and $m \geq 1$. In dimensional terms, the shear-rate viscosity, say, $\eta_{0}$ is higher that the infinite shear-rate viscosity, say, $\eta_{\infty}$. The parameter $m$ gives the ratio, $m=\eta_{0} / \eta_{\infty}$. Taking $m=1$ leads to Newtonian viscosity.

\section{Viscoelastic Fluids}

Viscoelastic fluids derive from either polymer solutions (part solvent and part polymeric) or polymer melts (wholly polymeric with no solvent component) and hence their total viscosity $\left(\eta_{T}\right)$ would comprise of a combination of solvent and polymeric contributions,

$$
\eta_{T}=\eta_{s}+\eta_{p}
$$

The parameter $\beta$ defines as the ratio of polymer to total viscosity, $\beta=\eta_{p} / \eta_{T}$. As with Newtonian fluids, the viscosity of ordinary viscoelastic fluids does not depend on prevailing shear-rate. This leads to,

$$
\begin{aligned}
& \eta_{s}(T)=(1-\beta) \mu(T), \\
& \eta_{p}(T)=\beta \mu(T) .
\end{aligned}
$$

Newtonian viscosity is recovered if $\beta=0$. Taking $\beta=1$ would model the viscosity of a polymer melt. Values of $\beta$ in the range $0<\beta<1$ therefore correspond to the viscosities of polymer solutions.

\section{Generalized Viscoelastic Fluids}

The Generalized Viscoelastic Fluids derive from adding shear-rate dependence to the polymer viscosity $\left(\eta_{p}\right)$. It is noted that the solvent viscosity is essentially always synonymous with Newtonian viscosity and hence can never be shear-rate dependent. The total viscosity for a Generalized Viscoelastic Fluid is therefore,

$$
\eta_{T}(\dot{\gamma}, T)=\eta_{s}(T)+\eta_{p}(\dot{\gamma}, T)
$$

The shear-thinning Carreau model is also employed for shear-rate dependence of viscosity and hence,

$$
\begin{aligned}
\eta_{s}(T) & =\left(1-\frac{\beta}{m}\right) \mu(T), \\
\eta_{p}(\dot{\gamma}, T) & =\frac{\beta}{m}\left[1+(m-1)\left(1+(\lambda \dot{\gamma})^{2}\right)^{\frac{n-1}{2}}\right] \mu(T) .
\end{aligned}
$$

where $m \geq 1$ and $0 \leq n \leq 1$.

Taking $m=1$ reduces the Generalized Viscoelastic Fluid viscosity model to that of an ordinary Viscoelastic Fluid. Taking $\beta=m$ reduces the Generalized Viscoelastic Fluid viscosity model to that of a Generalized Newtonian Fluid. Taking $\beta=0$ reduces the Generalized Viscoelastic Fluid viscosity model to that of a Newtonian fluid. Equations (18) and (19) therefore represent the most general viscosity constitutive model. 


\section{Initial and Boundary Conditions}

Equations (1-4) are solved subject to zero initial conditions,

$$
u(0, y)=0, \quad T(0, y)=0, \quad \boldsymbol{\tau}(0, y)=\mathbf{0}, \quad 0 \leq y \leq 1 .
$$

The velocity and temperature boundary conditions arise from no-slip velocity at the walls and uniform wall temperature,

$$
u(t, 0)=0, \quad T(t, 0)=1, \quad u(t, 1)=1, \quad T(t, 1)=1, \quad t \geq 0 .
$$

The boundary conditions for the extra stress are rebuilt from the main flow, [20].

\section{Numerical Solution}

The governing equations are solved using a robust and efficient semi-implicit finite difference algorithm given in $[14,15,20]$. Implicit terms are taken at the intermediate time level $(n+\zeta)$ where $0 \leq \zeta \leq 1$. The choice $\zeta=1$ admits larger time steps and leads to very rapid convergence and hence will be used in this article. The discretization of the governing equations in space is based on a uniform Cartesian grid. Both the second and first spatial derivatives are approximated using second-order central differences. The equations corresponding to the boundary points are modified as necessary to incorporate the boundary conditions. The semi-implicit algorithm for the velocity component is,

$$
\operatorname{Re} \frac{u^{(n+1)}-u^{(n)}}{\Delta t}=\frac{\partial}{\partial y} \tau_{12}^{(n)}+\left(\eta_{s}^{(n)} \frac{\partial^{2}}{\partial y^{2}} u^{(n+\zeta)}+\frac{\partial}{\partial y} u^{(n)} \frac{\partial}{\partial y} \eta_{s}^{(n)}\right),
$$

where

$$
u^{(n+\zeta)}=\zeta u^{(n+1)}+(1-\zeta) u^{(n)} .
$$

The equation for the new velocity at the next time step, $u^{(n+1)}$, becomes,

$$
\begin{aligned}
& -r_{1} u_{j-1}^{(n+1)}+\left(\operatorname{Re}+2 r_{1}\right) u_{j}^{(n+1)}-r_{1} u_{j+1}^{(n+1)}=\operatorname{Re} u_{j}^{(n)}+(1-\zeta) \Delta t \eta_{s}^{(n)} \frac{\partial^{2}}{\partial y^{2}} u^{(n)} \\
& +\Delta t \frac{\partial}{\partial y} \tau_{12}^{(n)}+(1-\beta) \Delta t \frac{\partial}{\partial y} u^{(n)} \frac{\partial}{\partial y} \eta_{s}^{(n)},
\end{aligned}
$$

where

$$
r_{1}=\zeta \eta_{s}^{(n)} \frac{\Delta t}{\Delta y^{2}}
$$

The solution procedure for $u^{(n+1)}$ reduces to the numerically stable and efficient process of inversion of diagonally dominant tri-diagonal matrices. This is a huge advantage over a fully implicit scheme. The semi-implicit algorithm for the temperature equation is similarly obtained,

$$
\operatorname{Re} \operatorname{Pr} \frac{\partial T}{\partial t}=\frac{\partial^{2}}{\partial y^{2}} T^{(n+\zeta)}+\operatorname{Br} Q_{D}^{(n)}+\delta_{1} \exp \left(\frac{T^{(n)}}{1+\alpha T^{(n)}}\right) .
$$

The equation for the new temperature at the next time step, $T^{(n+1)}$, becomes,

$$
-r_{2} T_{j-1}^{(n+1)}+\left(\operatorname{Re} \operatorname{Pr}+2 r_{2}\right) T_{j}^{(n+1)}-r_{2} T_{j+1}^{(n+1)}=\operatorname{Re} \operatorname{Pr} T_{j}^{(n)}+(1-\zeta) \Delta t \frac{\partial^{2}}{\partial y^{2}} T^{(n)}
$$




$$
\begin{aligned}
& +\Delta t \operatorname{Br} \eta_{s}^{(n)}\left(\frac{\partial}{\partial y} u^{(n)}\right)^{2}+\delta_{1} \exp \left(\frac{T^{(n)}}{1+\alpha T^{(n)}}\right)+\Delta t \operatorname{Br} \gamma \tau_{12}^{(n)} \frac{\partial}{\partial y} u^{(n)} \\
& +(1-\gamma) \Delta t \operatorname{Br} \frac{\hat{G}}{2 \operatorname{De} \bar{\lambda}(T)}\left(I_{1}+\operatorname{Tr}\left(\boldsymbol{b}^{-1}\right)-6\right),
\end{aligned}
$$

where

$$
r_{2}=\zeta \frac{\Delta t}{\Delta y^{2}}
$$

As with the velocity equation, the solution procedure for the temperature reduces to inversion of a diagonally dominant tri-diagonal matrix. The tri-diagonal matrix for the temperature algorithm in fact remains constant in time.

The constitutive equations for the extra stress, Eq. (4), are solved analogously via semiimplicit algorithms,

$$
\boldsymbol{\tau}^{(n+\zeta)}+\operatorname{De} \bar{\lambda}^{(n)} \frac{\boldsymbol{\tau}^{(n+1)}-\boldsymbol{\tau}^{(n)}}{\Delta t}=\text { explicit terms. }
$$

The equations for the extra stress components at the next time level, $\tau_{11}^{(n+1)}, \tau_{12}^{(n+1)}$ and $\tau_{22}^{(n+1)}$ are thus all of the form,

$$
\left(\mathrm{De} \bar{\lambda}^{(n)}+\zeta \Delta t\right) \boldsymbol{\tau}^{(n+1)}=\text { explicit terms. }
$$

The explicit terms for the equations connected to each of the extra stress components, $\tau_{11}$, $\tau_{12}$ and $\tau_{22}$ are respectively,

$$
\begin{gathered}
{\left[\operatorname{De} \bar{\lambda}^{(n)}-(1-\zeta) \Delta t\right] \tau_{11}^{(n)}+\Delta t \operatorname{De} \bar{\lambda}^{(n)}\left[\tau_{12}^{(n)} \frac{\partial}{\partial y} u^{(n)}+\tau_{11}^{(n)} \frac{\partial}{\partial y} \log \left(1+\alpha T^{(n)}\right)\right]} \\
{\left[\operatorname{De} \bar{\lambda}^{(n)}-(1-\zeta) \Delta t\right] \tau_{12}^{(n)}+\Delta t \operatorname{De} \bar{\lambda}^{(n)}\left[\tau_{22}^{(n)} \frac{\partial}{\partial y} u^{(n)}+\tau_{12}^{(n)} \frac{\partial}{\partial y} \log \left(1+\alpha T^{(n)}\right)\right]} \\
+\Delta t \eta_{p} \frac{\partial}{\partial y} u^{(n)} \\
{\left[\operatorname{De} \bar{\lambda}^{(n)}-(1-\zeta) \Delta t\right] \tau_{22}^{(n)}+\Delta t \operatorname{De} \bar{\lambda}^{(n)} \tau_{22}^{(n)} \frac{\partial}{\partial y} \log \left(1+\alpha T^{(n)}\right)}
\end{gathered}
$$

The numerical solution procedures therefore reduce to direct algebraic manipulation.

\section{Results}

In addition to the velocity $(u)$, temperature $(T)$, and extra-stress components $\left(\tau_{11}, \tau_{12}, \tau_{22}\right)$, results will also be given for the normal stress difference, $N_{1}$,

$$
N_{1}=\tau_{11}-\tau_{22} \text {. }
$$

Results of $N_{1}$ are of crucial importance in checking the physical correctness of the viscoelastic computations. In particular, it must always be the case that,

$$
N_{1} \geq 0 \quad \forall t \geq 0, \text { and } \forall y: 0 \leq y \leq 1 .
$$

Unless the context otherwise dictates, the embedded parameters will be assumed to take the following values,

$$
\alpha=0.1, \operatorname{Br}=1, \operatorname{Re}=1, \operatorname{Pr}=1, \operatorname{De}=3, \gamma=0.5, \beta=0.6, \Delta y=0.01,
$$



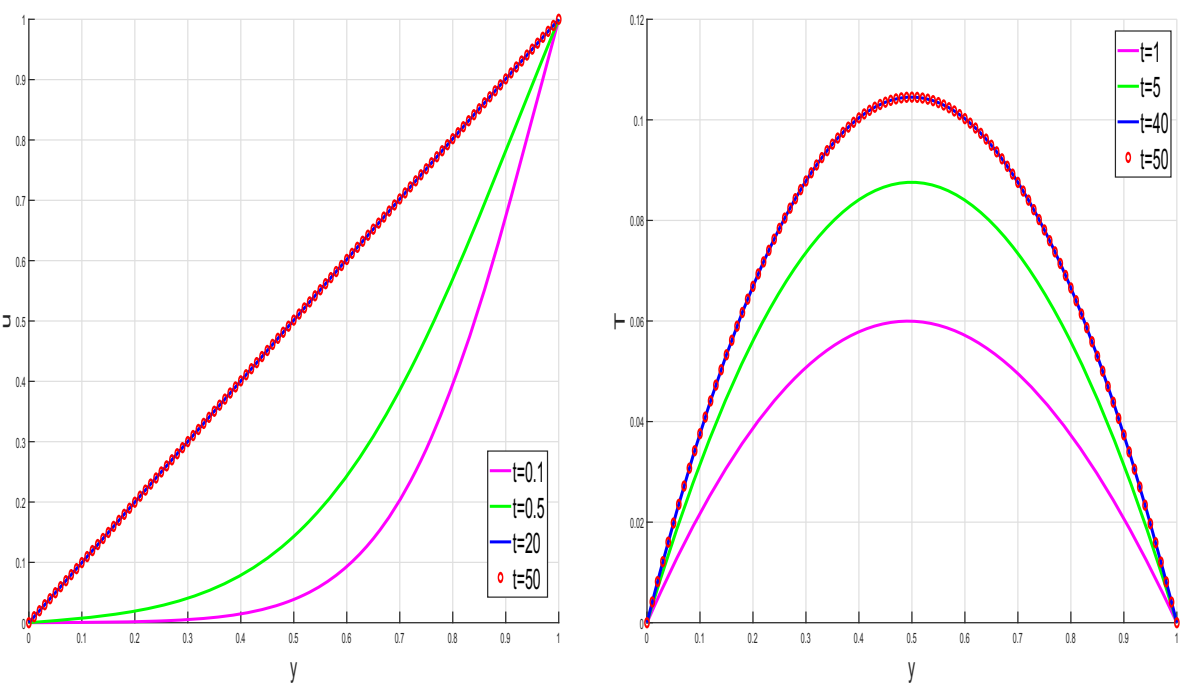

Fig. 2 Development of steady velocity and temperature profiles

$$
\Delta t=0.01, t=50, \hat{G}=10^{-3}, m=2, \delta_{1}=0.1, \lambda=0.1, n=0.5, \zeta=1 .
$$

\section{Development of Steady Solutions}

Figures 2-5 give the time development of solutions to steady state. The time evolution of solutions shows, as expected, that the diagonal stress component $\tau_{22}$ remains at zero and hence also that the first normal stress difference $N_{1}=\tau_{11}-\tau_{22} \equiv \tau_{11}$. This, in fact, is the case in all the succeeding computations. These predictable graphical profiles of both $\tau_{22}$ and $N_{1}$ will therefore be omitted in the subsequent graphical analysis. The steady velocity also reproduces the same linear graph and hence this predictable profile will also be omitted from the subsequent analysis.

Except for the velocity profile, whose maximum is always one, obtained at the boundary $(u(t, 1) \equiv 1)$, Fig. 5 plots the maximum flow quantities at steady state, $t=50$. For the velocity profile, the value at the center of the channel, $u(t, 0.5)$, will be plotted.

\section{Temporal and Spatial Convergence}

Figures 6 and 7 demonstrate the convergence of the algorithms with respect to both time step size and mesh size. It is observed that the solutions are independent of the chosen time-step size for a wide range of time-step sizes and also similarly that the solutions are independent of the mesh size even for moderate mesh sizes.

\section{Parameter Dependence of Solutions}

In this section, attention is focused on the sensitivity of the solutions to variations in some representative parameters. The sensitivity of solutions to variations in the Frank-Kamenetskii parameter is shown in Figs. 8 and 9 

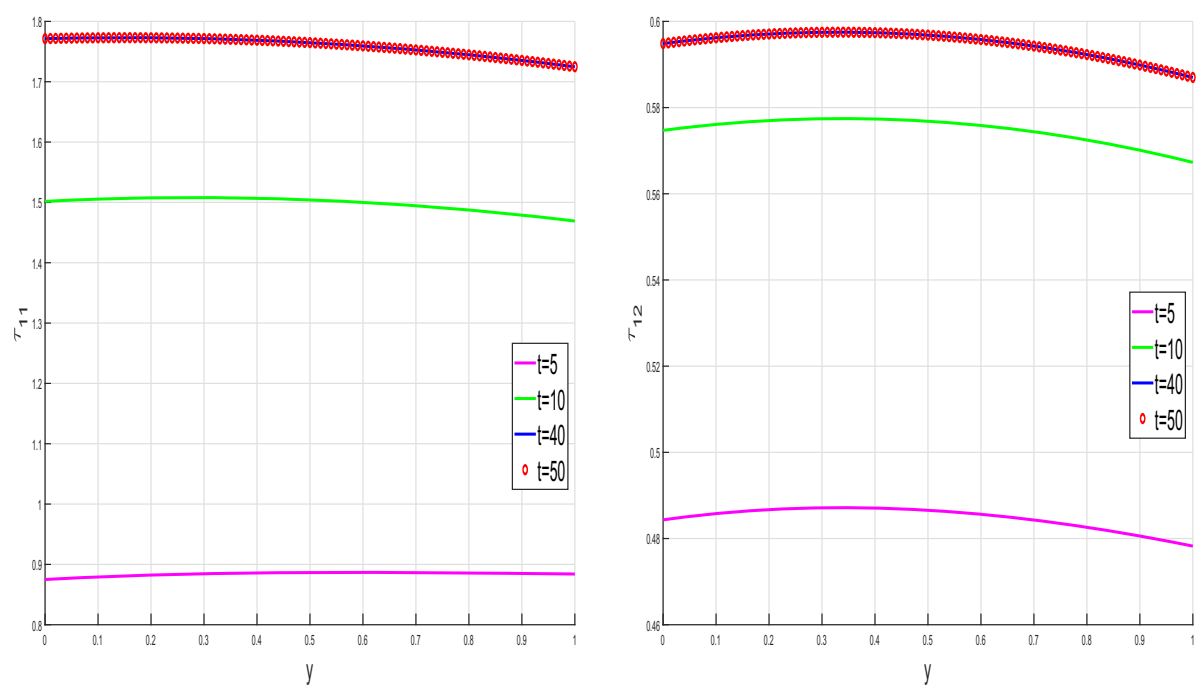

Fig. 3 Development of steady $\tau_{11}$ and $\tau_{12}$ profiles
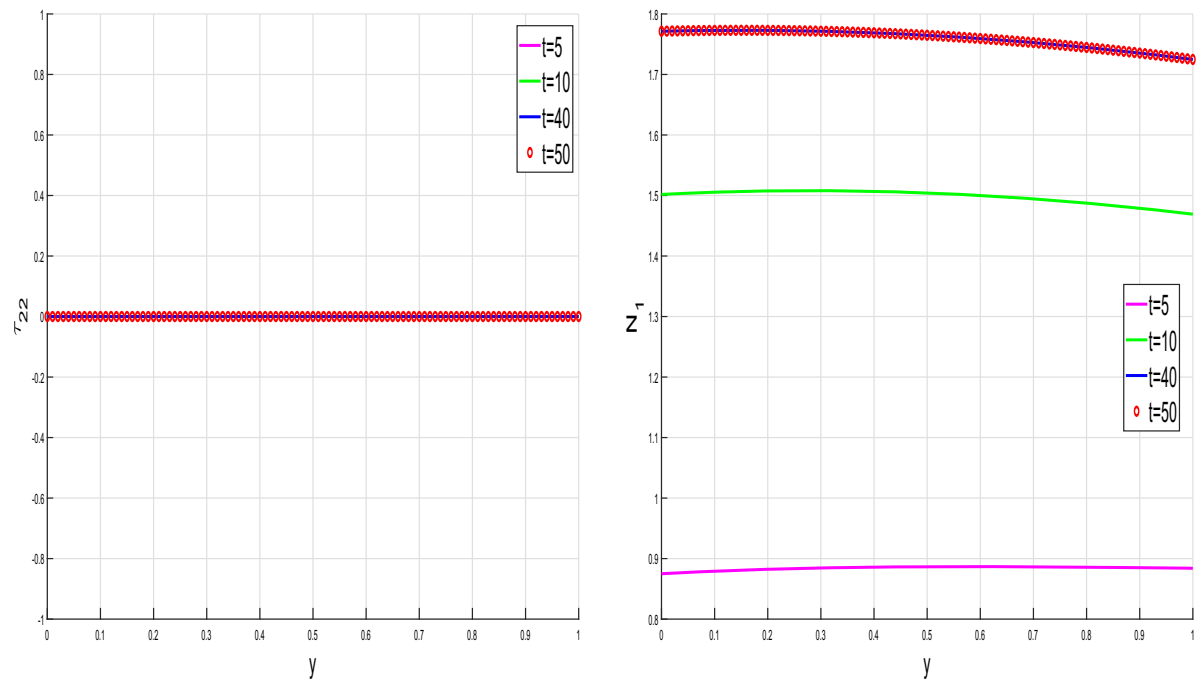

Fig. 4 Development of steady $\tau_{22}$ and $N_{1}$ profiles

In Figs. 8 and 9, save for the varying values of $\delta_{1}$ as reflected, all other parameters are otherwise given in Eq. (30). This will be the case for the remainder of the article, the variable parameter(s) will be clearly given by the context but otherwise all other parameter values will be as in Eq. (30). The parameter values given in Eq. (30) otherwise represent the most general fluid type, i.e. the Generalized Viscoelastic Fluid.

As expected, Fig. 9 shows that the fluid temperature increases significantly with increases in the reaction parameter $\delta_{1}$. The dependence of the fluid viscoelasticity with increasing $\delta_{1}$ behaves oppositely to the temperature dependence. The polymeric stresses decrease with increasing $\delta_{1}$. 


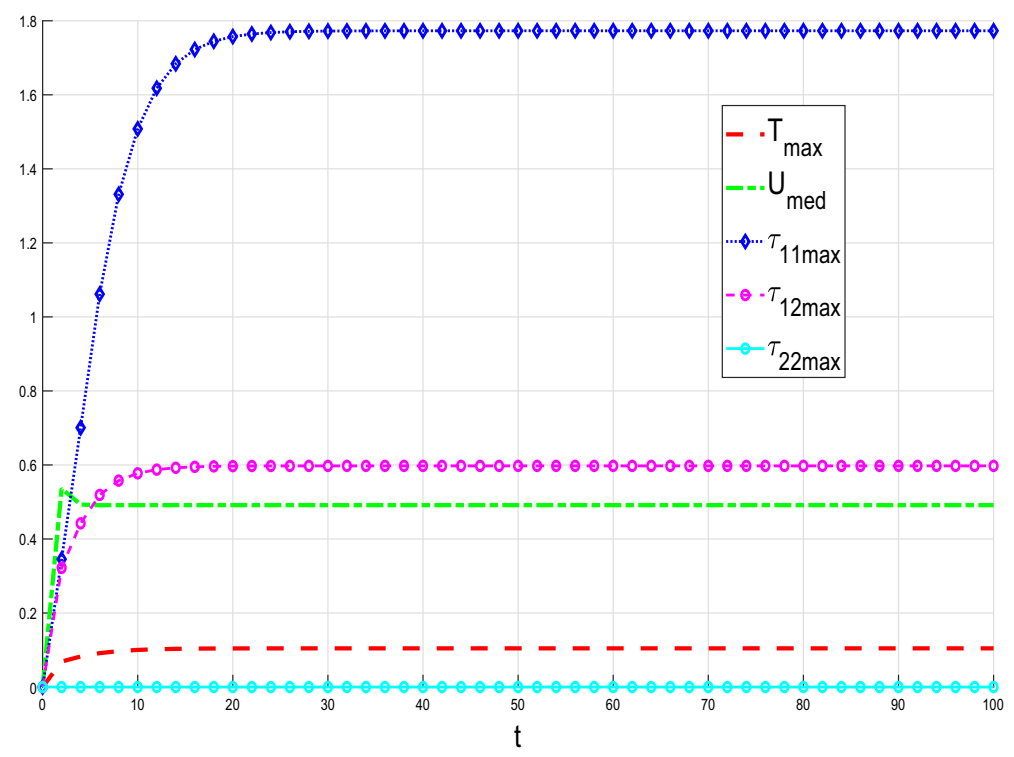

Fig. 5 Development of steady maximum flow quantities

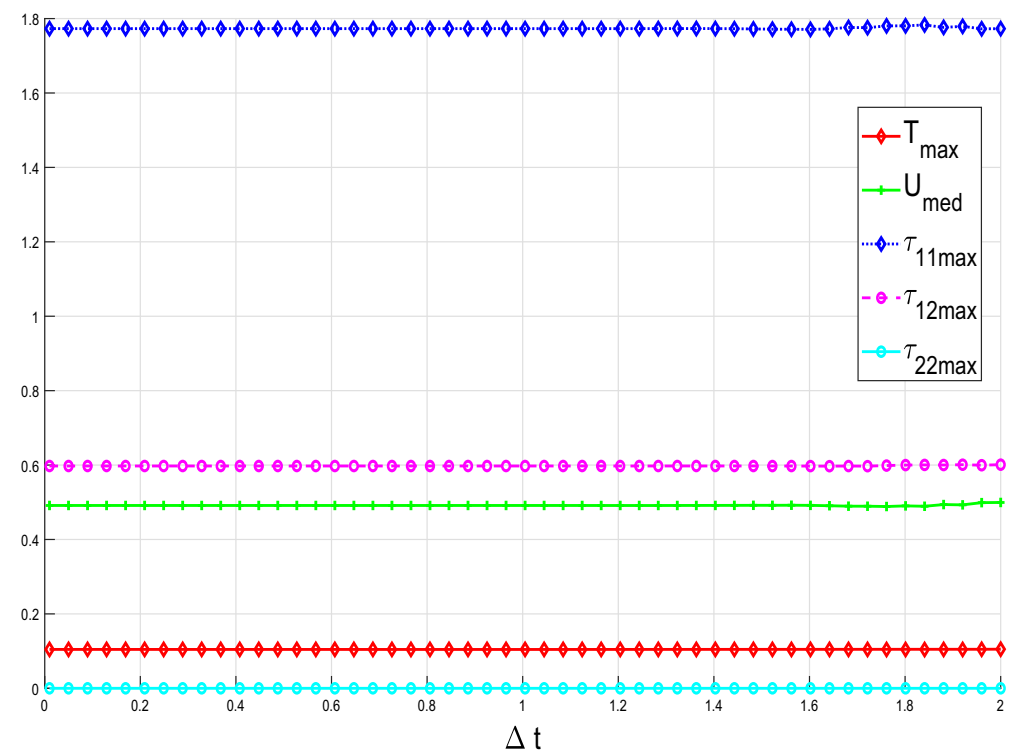

Fig. 6 Dependence of solutions on time step size

Being an exothermic reaction parameter, the values of $\delta_{1}$ need to be carefully controlled to avoid finite time temperature blow up. Figure 10 demonstrates the blow up of temperature for the different fluid types. It is observed that the Oldroyd-B viscoelastic fluids $(m=1)$ are the least susceptible to blow up, followed by the Generalized Oldroyd-B fluids, and 


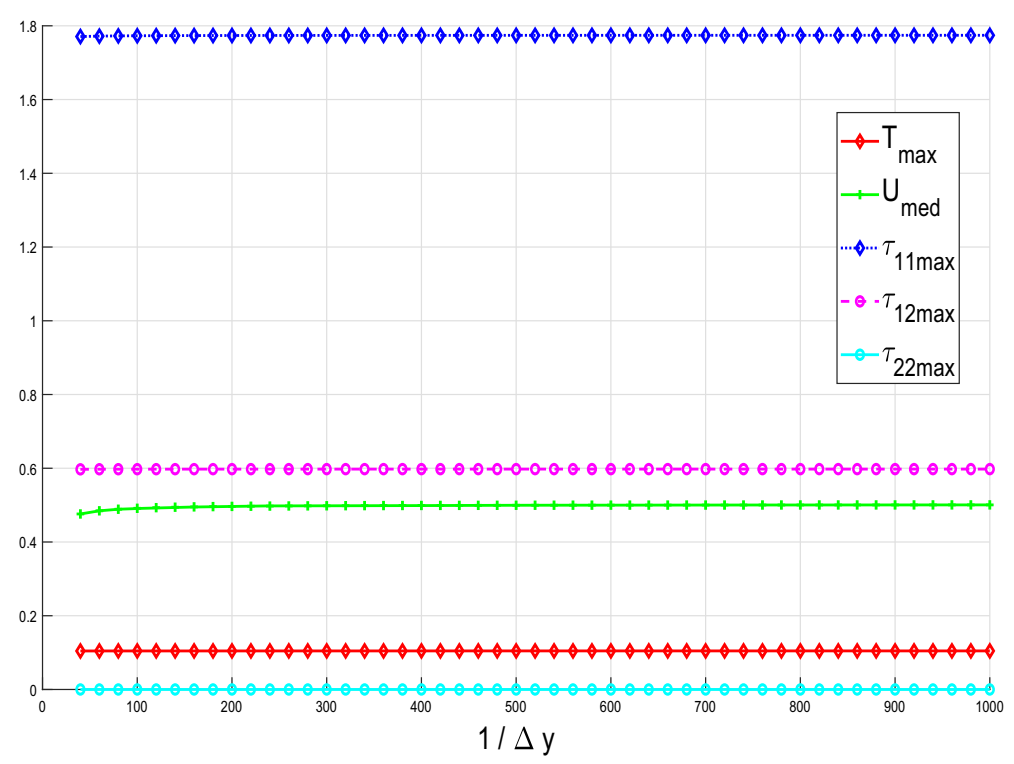

Fig. 7 Dependence of solutions on mesh size
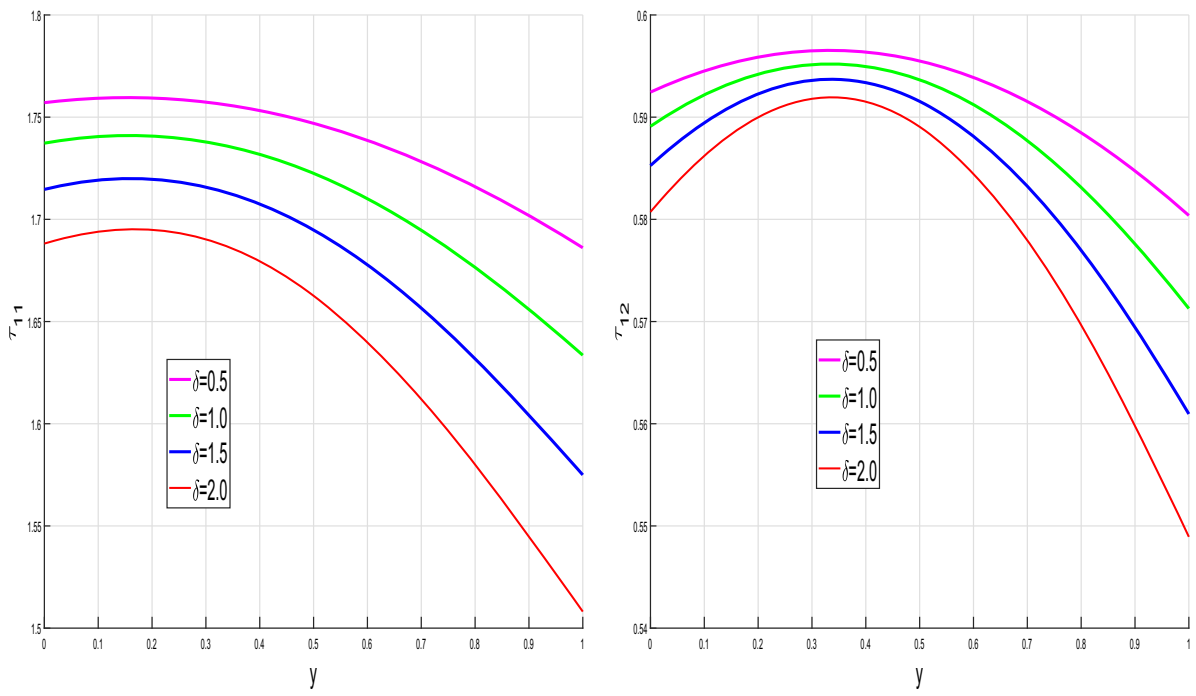

Fig. 8 Sensitivity of $\tau_{11}$ and $\tau_{12}$ to $\delta_{1}$

then by the Newtonian fluids ( $m=1, \beta=0$, De $=0$ ). The Generalized Newtonian Fluids $(\beta=0, \mathrm{De}=0)$ are most susceptible to blow up.

The shear-thinning characteristics of the Generalized Fluid Models therefore make them least resistant to large temperature increases and hence makes them more susceptible to finite time temperature blow up. The observations in Fig. 10 are confirmed in the exploratory study [12] as well as in the previous investigations [2]. 


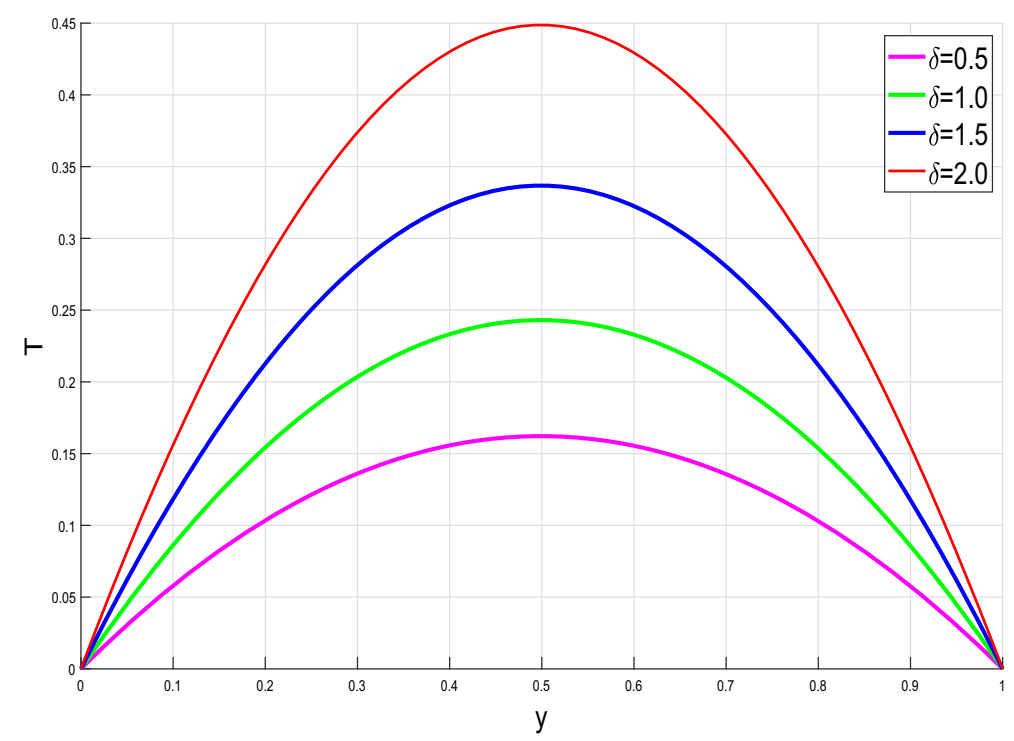

Fig. 9 Sensitivity of fluid temperature to $\delta_{1}$

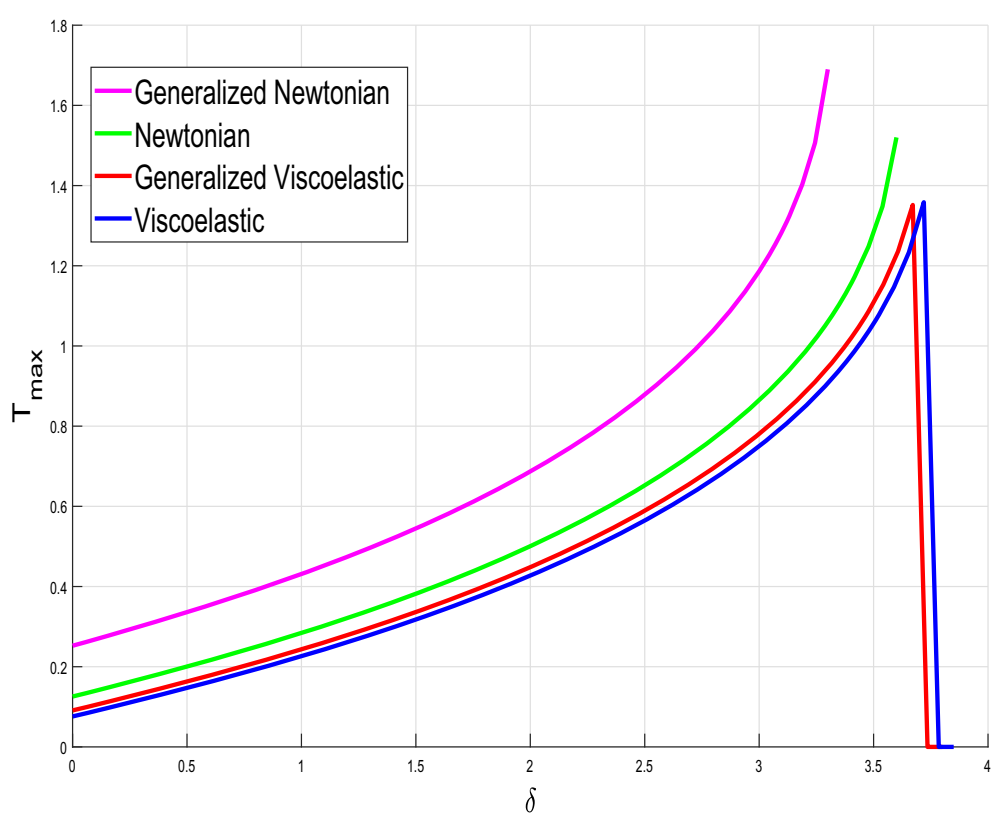

Fig. 10 Blow up of temperature with $\delta_{1}$ for various fluid types

Figures 11 and 12 illustrate the sensitivity of solutions to variations in the polymerviscosity parameter, $\beta$.

As expected, an increase in $\beta$ correspondingly increases the polymer stresses as well as the normal stress differences. This in turn acts to resist heat build-up and hence the observed reduction in temperature with increasing $\beta$. As illustrated in Figs. 11 and 12, the temperature 

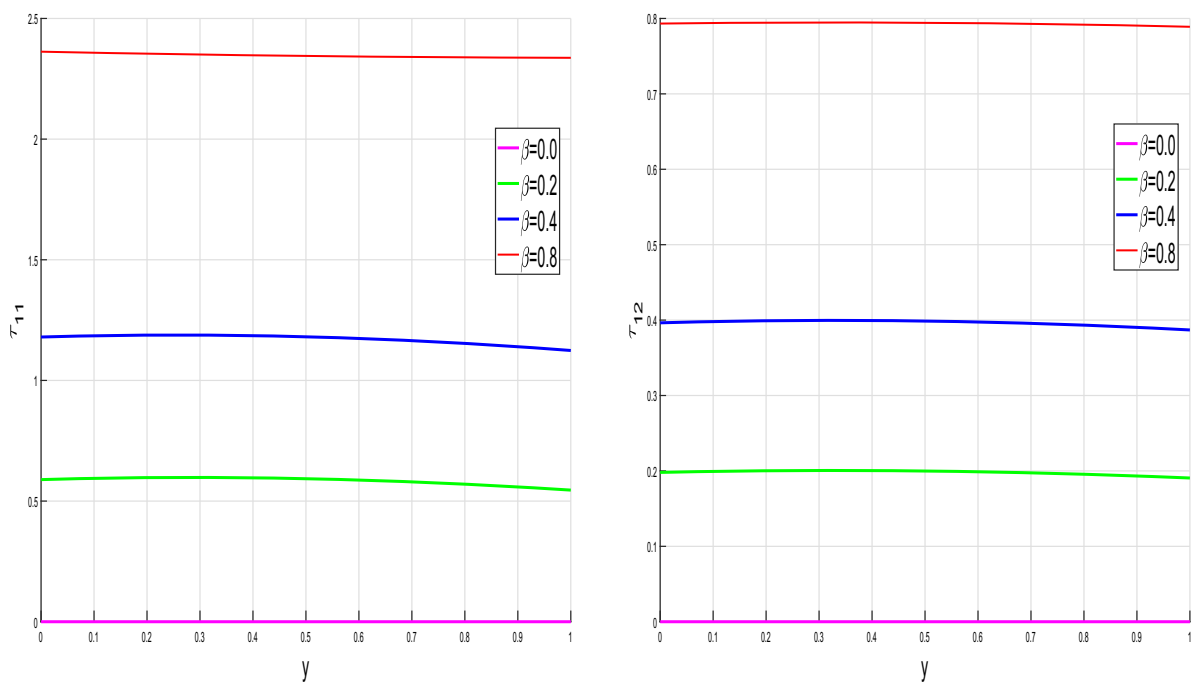

Fig. 11 Sensitivity of $\tau_{11}$ and $\tau_{12}$ to $\beta$
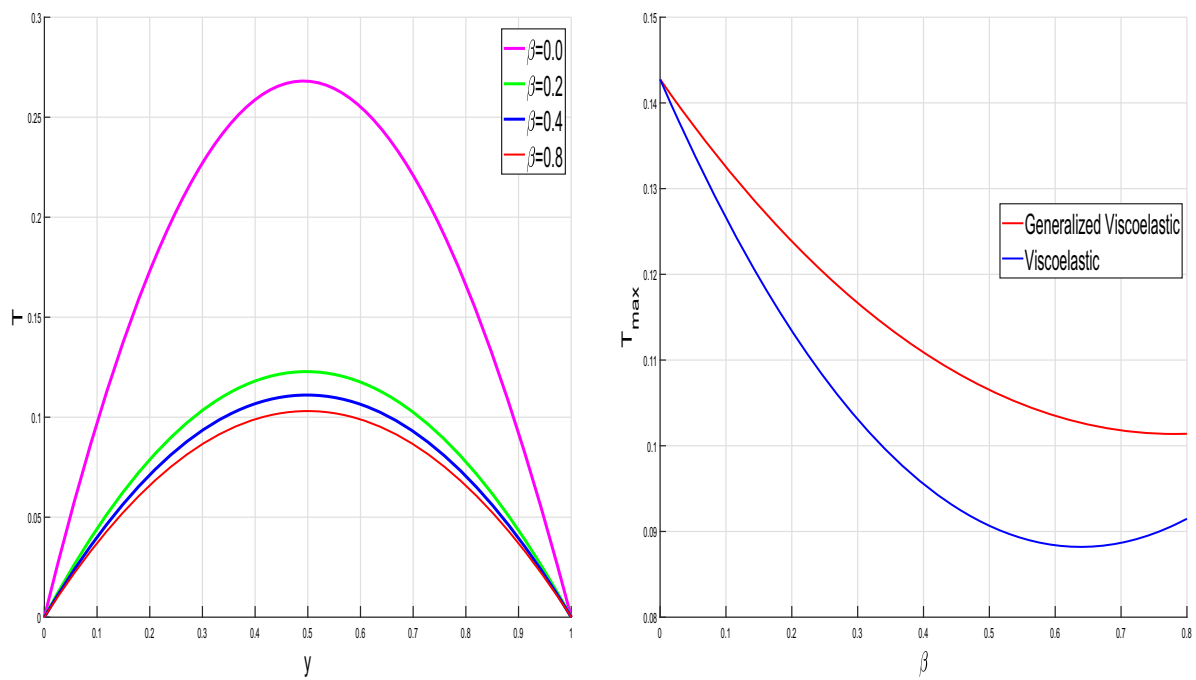

Fig. 12 Sensitivity of fluid temperature to $\beta$

reduction might occur in a non-monotonic way in which the minimum temperature would be achieved for a polymer solution, $0<\beta<1$, rather than for a polymer melt, $\beta=1$. Whichever the case might be, the highest temperatures are recorded for in-elastic fluids, $\beta=0$. Lower temperatures are otherwise recorded for the Oldroyd-B fluids $(m=1)$ compared to the Generalized Oldroyd-B fluids.

Figures 13 and 14 illustrate the sensitivity of solutions to variations in the Brinkman number, $\mathrm{Br}$.

The dependence of the flow quantities with variations in the Brinkman number, $\mathrm{Br}$, is analogous to that with respect to the reaction parameter, $\delta_{1}$, the main difference being the 

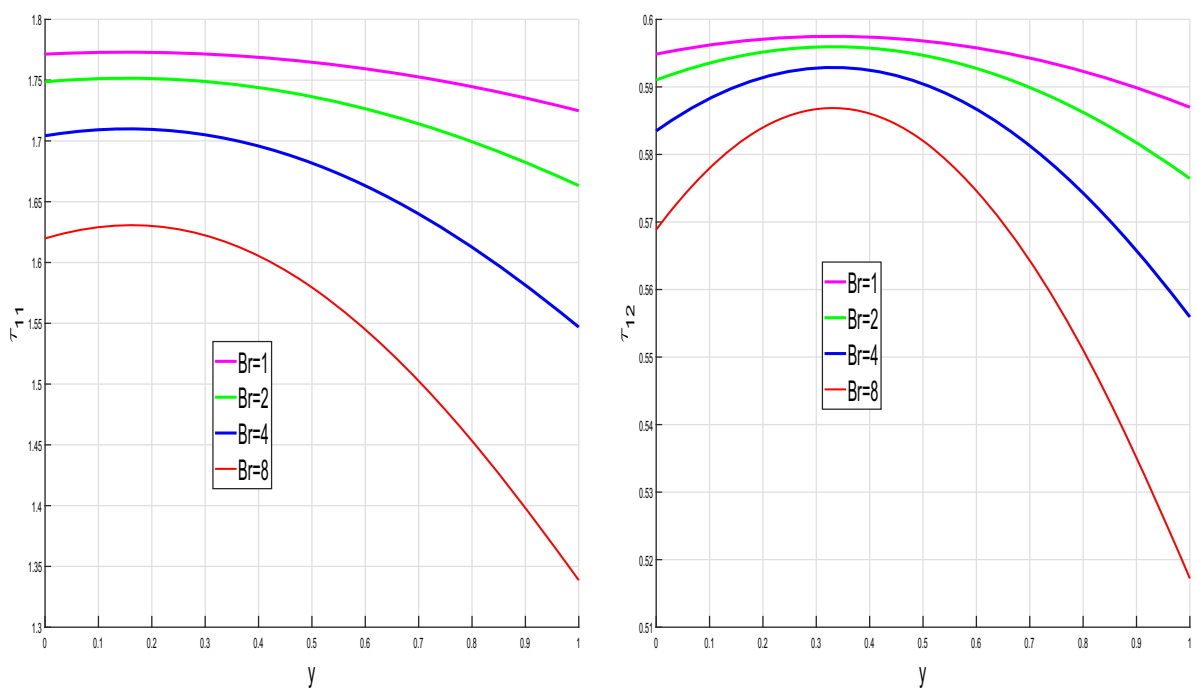

Fig. 13 Sensitivity of $\tau_{11}$ and $\tau_{12}$ to $\mathrm{Br}$
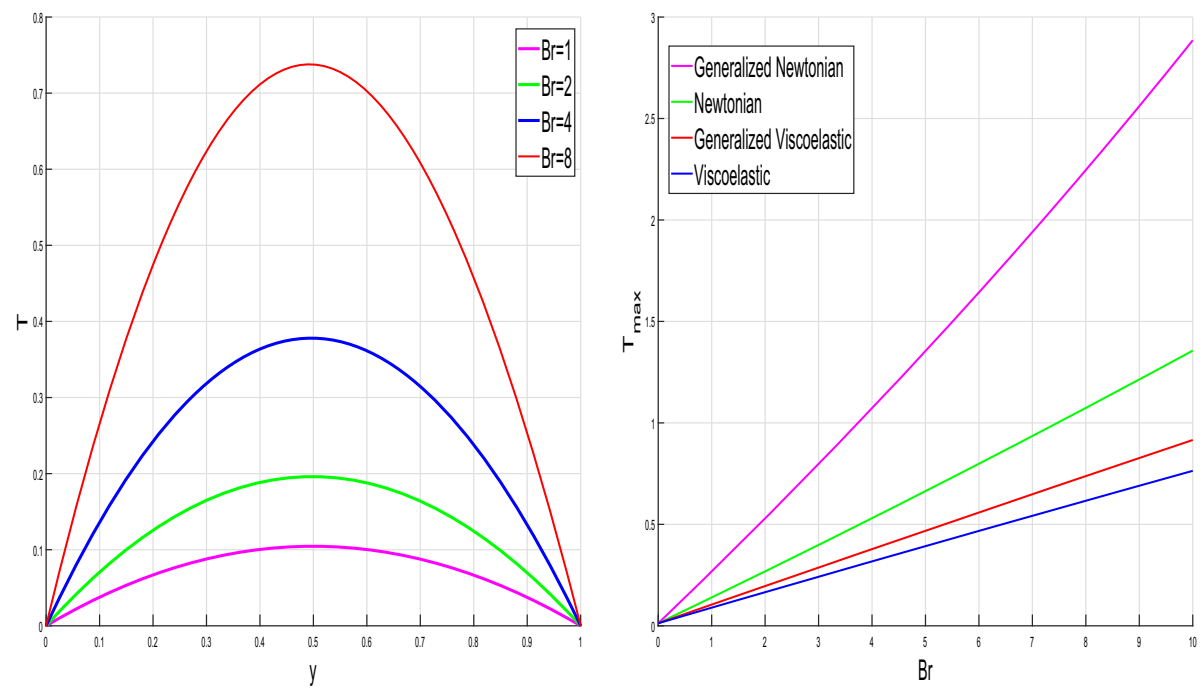

Fig. 14 Sensitivity of fluid temperature to $\mathrm{Br}$

now absence of thermal runaway phenomena due to linear rather than exponential temperature growth. As expected therefore, Fig. 9 shows that the fluid temperature increases roughly linearly with increases in the Brinkman number, $\mathrm{Br}$. The dependence of the fluid viscoelasticity with increasing Br behaves oppositely to the temperature dependence. The polymeric stresses decrease with increasing Br. It is also observed that the Oldroyd-B fluids $(m=1)$ are most resistant to temperature increases, followed by the Generalized Oldroyd-B fluids, and then by the Newtonian fluids $(m=1, \beta=0, \mathrm{De}=0)$, with the Generalized Newtonian Fluids $(\beta=0, \mathrm{De}=0$ ) offering least resistance to temperature increases. 

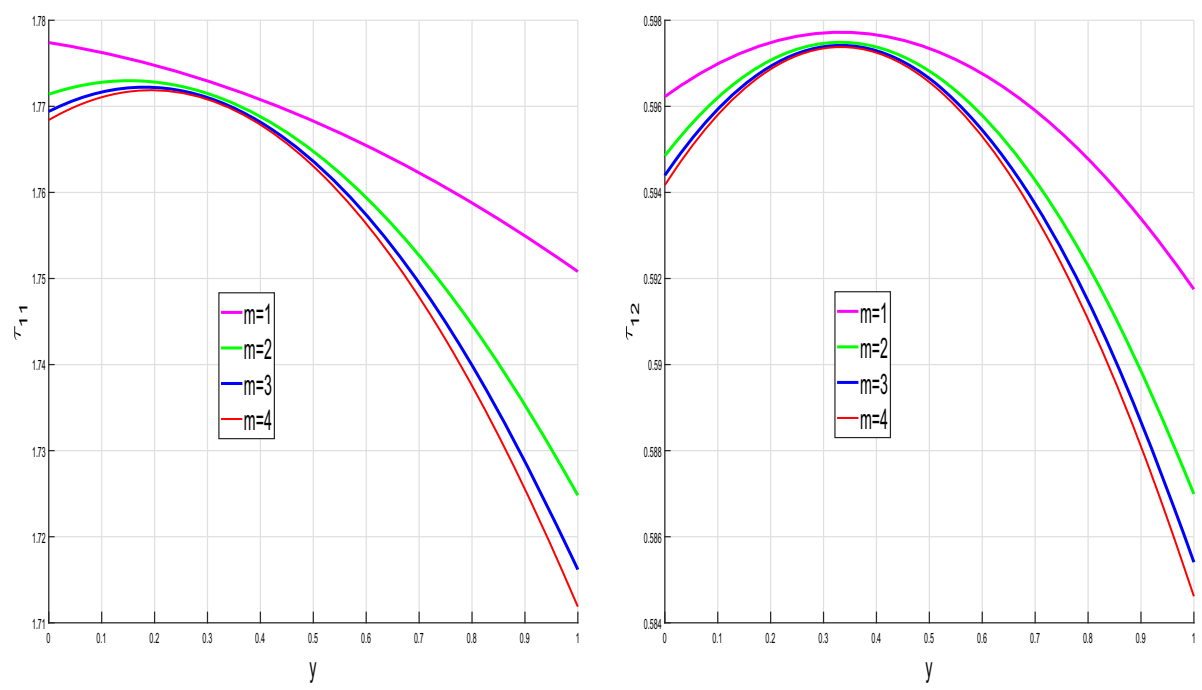

Fig. 15 Sensitivity of $\tau_{11}$ and $\tau_{12}$ to $m$
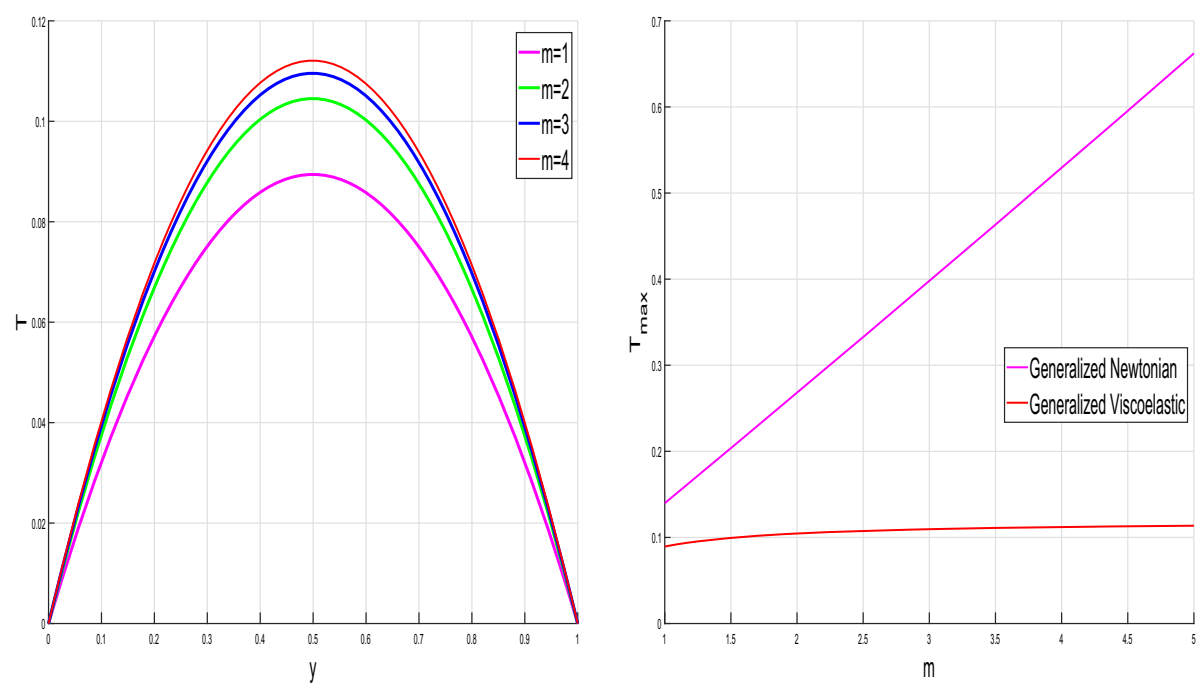

Fig. 16 Sensitivity of fluid temperature to $m$

Figures 15 and 16 illustrate the sensitivity of solutions to variations in the shear-thinning parameter, $m$.

As with the Brinkman number, $\mathrm{Br}$, and the reaction parameter, $\delta_{1}$, it is observed that the fluid temperature increases (but at most only linearly) with increases in the shear-thinning parameter, $m$. The dependence of the fluid viscoelasticity with increasing $m$ also behaves oppositely to the temperature dependence. The polymeric stresses decrease with increasing $m$. It is also observed that the Generalized Oldroyd-B fluids are most resistant to temperature increases as compared to the Generalized Newtonian Fluids $(\beta=0$, De $=0)$. 

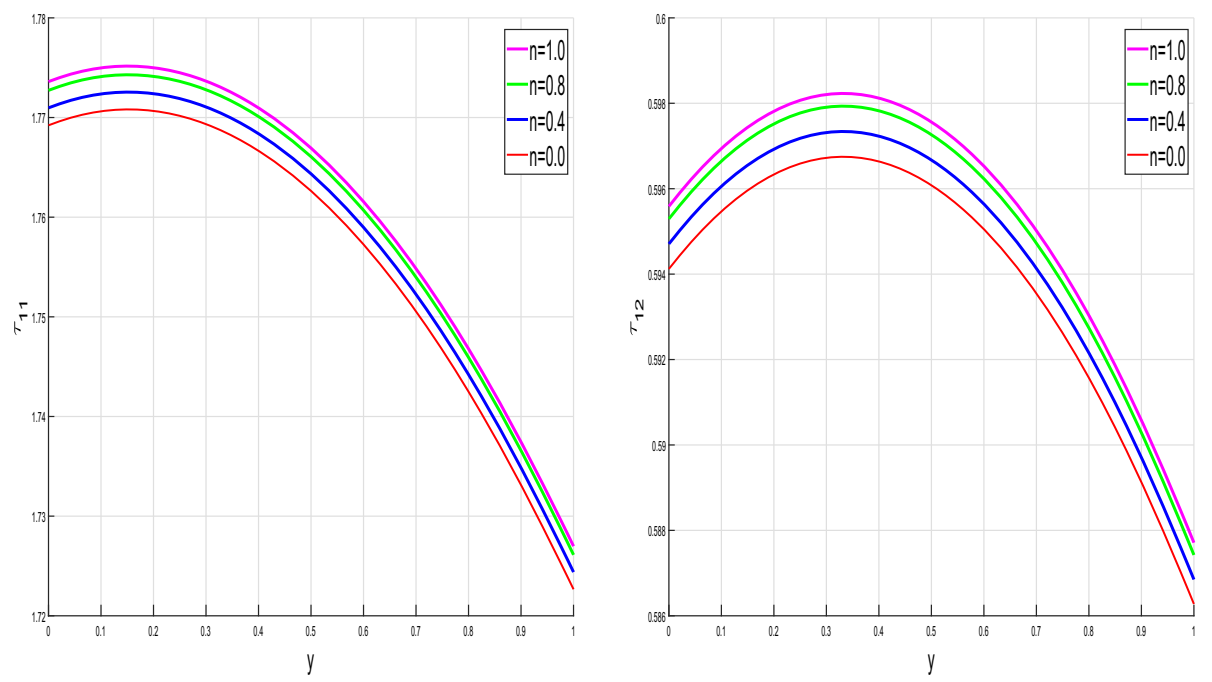

Fig. 17 Sensitivity of $\tau_{11}$ and $\tau_{12}$ to $n$
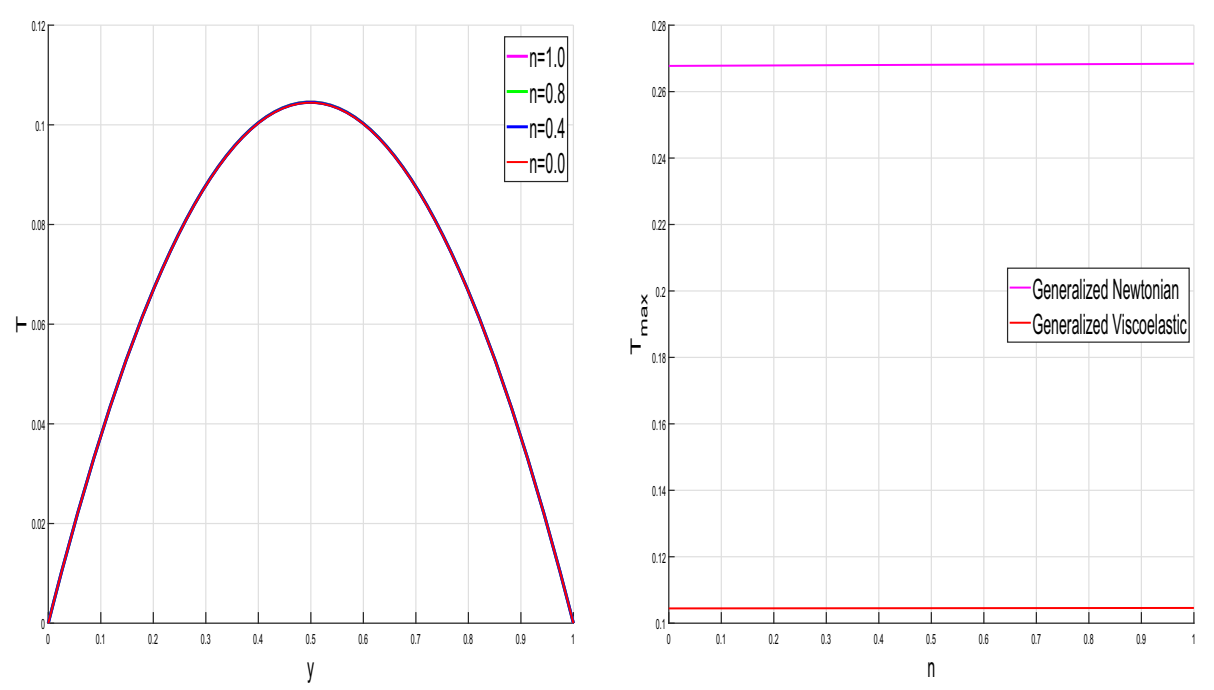

Fig. 18 Sensitivity of fluid temperature to $n$

Figures 17 and 18 illustrate the sensitivity of solutions to variations in the shear-thinning parameter, $n$.

The dependence of flow quantities on the shear-thinning parameter, $n$, is analogous to that with respect to the other shear-thinning parameter, $m$. The only difference being with regards to the magnitude of the increases/decreases of flow quantities. 


\section{Conclusion}

A comparative analysis of the response of the four broad categories of fluids to non-isothermal effects and exothermic reactions in simple shear flow is conducted. The four fluid categories of focus are the; Newtonian fluids; shear-thinning Generalized Newtonian Fluids; Viscoelastic fluids; and shear-thinning Generalized Viscoelastic Fluids. The Carreau model is employed for the shear-thinning viscosity and the Oldroyd-B model is used to describe the viscoelastic fluids. The results demonstrate that the Generalized Newtonian Fluids are most susceptible to thermal runaway phenomena, followed by Newtonian fluids, which are in turn followed by the Generalized Viscoelastic Fluids, with the Viscoelastic fluids recording the least susceptibility to thermal runaway. These result are of fundamental importance to industrial and biological applications in which heat generation minimization is important, such as in heat exchangers, in lubrication, and in internal medicine.

In general, it is observed that the fluid temperature (respectively the polymer stresses) increases (respectively decrease) with increasing shear-thinning character. It is also observed that the fluid temperature (respectively the polymer stresses) decreases (respectively increase) with increasing polymeric character.

Acknowledgements Tiri Chinyoka is grateful for the exploratory work that was conducted by T. Hlope in [12], which work laid the foundation of this research.

Author Contributions Tiri Chinyoka exclusively contributed to all aspects of this research article. All background work and references have been acknowledged as necessary.

\section{Declarations}

Conflict of interests The author declares that they have no conflict of interests.

Ethical Statement The author confirms that this research article conforms with the relevant ethics in research.

\section{References}

1. Chinyoka, T.: Viscoelastic effects in double-pipe single-pass counterflow heat exchangers. Int. J. Numer. Meth. Fluids 59(6), 677-690 (2009)

2. Chinyoka, T.: Computational dynamics of a thermally decomposable viscoelastic lubricant under shear. J. Fluids Eng. 130(12), 121201 (2008)

3. Deville, M., Gatski, T.B.: Mathematical Modeling for Complex Fluids and Flows. Springer, Berlin (2012)

4. Tanner, R.I.: Engineering Rheology, vol. 52, 2nd edn. Oxford University Press, Oxford (2000)

5. Larson, R.G.: The Structure and Rheology of Complex Fluids, vol. 150. Oxford University Press, New York (1999)

6. Tanner, R.I., Walters, K.: Rheology: An Historical Perspective, vol. 7. Elsevier, Amsterdam (1998)

7. Carreau, P.J., De Kee, D.C.R., Chhabra, R.P.: Rheology of Polymeric Systems, Principles and Applications. American Institute of Chemical EngineersHanser, New York (1997)

8. Barnes, H.A., Hutton, J.F., Walters, K.: An Introduction to Rheology, vol. 3. Elsevier, Amsterdam (1989)

9. Bird, R.B., Armstrong, R.C., Hassager, O.: Dynamics of Polymeric Liquids, Fluid Mechanics, vol. 1, 2nd edn. Wiley, Hoboken (1987)

10. Schowalter, W.R.: Mechanics of Non-Newtonian Fluids. Pergamon Press, Oxford (1978)

11. Bird, R.B.: Useful non-Newtonian models. Annu. Rev. Fluid Mech. 8, 13-34 (1976)

12. Hlope, T., Chinyoka, T.: Comparative Study of the Thermal Loading Properties of Newtonian and NonNewtonian Fluids in Shear Flow, Master's Thesis, African Institute for Mathematical Sciences, AIMS Africa, Muizenberg, South Africa (2018)

13. Ali, U., Rehman, K.U., Alshomrani, A.S., Malik, M.Y.: Thermal and concentration aspects in Carreau viscosity model via wedge. Case Stud. Thermal Eng. 12, 126-133 (2018) 
14. Chinyoka, T., Makinde, O.D.: Numerical analysis of the transient and non-isothermal channel flow of a third-grade fluid with convective cooling. Eng Trans (2020)

15. Ireka, I.E., Chinyoka, T.: Analysis of shear banding phenomena in non-isothermal flow of fluids governed by the diffusive Johnson-Segalman model. Appl. Math. Model. 40(5-6), 3843-3859 (2016)

16. Chinyoka, T.: Suction-injection control of shear banding in non-isothermal and exothermic channel flow of Johnson-Segalman liquids. J. Fluids Eng. 133(7), 071205 (2011)

17. Crochet, M.J., Davies, A.R., Walters, K.: Numerical Simulation of Non-Newtonian Flow, vol. 1. Elsevier, Amsterdam (2012)

18. Khan, M., Sardar, H., Gulzar, M.M., Alshomrani, A.S.: On multiple solutions of non-Newtonian Carreau fluid flow over an inclined shrinking sheet. Results Phys. 8, 926-932 (2018)

19. Rehman, K.U., Malik, M.Y., Khan, A.A., Zehra, I., Zahri, M., Tahir, M.: Numerical solution of chemically reactive non-Newtonian fluid flow: dual stratification. Eur. Phys. J. Plus 132, 550 (2017)

20. Chinyoka, T., Goqo, S.P., Olajuwon, B.I.: Computational analysis of gravity driven flow of a variable viscosity viscoelastic fluid down an inclined plane. Comput. Fluids 84, 315-326 (2013)

21. Makinde, O.D.: Thermal stability of a reactive viscous flow through a porous-saturated channel with convective boundary conditions. Appl. Therm. Eng. 29(8/9), 1773-1777 (2009)

22. Makinde, O.D., Chinyoka, T.: Numerical study of unsteady hydromagnetic Generalized Couette flow of a reactive third-grade fluid with asymmetric convective cooling. Comput. Math. Appl. 61(4), 1167-1179 (2011)

23. Koriko, O.K., Animasaun, I.L., Mahanthesh, B., Saleem, S., Sarojamma, G., Sivaraj, R.: Heat transfer in the flow of blood-gold Carreau nanofluid induced by partial slip and buoyancy. Heat Transfer-Asian Res. 47, 806-823 (2018)

24. Makinde, O.D., Omojola, M.T., Mahanthesh, B., Alao, F.I., Adegbie, K.S., Animasaun, I.L., Wakif, A., Sivaraj, R., Tshehla, M.S.: Significance of buoyancy, velocity index and thickness of an upper horizontal surface of a paraboloid of revolution: the case of non-Newtonian carreau fluid. Defect Diffusion. Forum 387, 550-561 (2018)

Publisher's Note Springer Nature remains neutral with regard to jurisdictional claims in published maps and institutional affiliations. 